Proyecciones Journal of Mathematics

Vol. 28, No 3, pp. 271-283, December 2009.

Universidad Católica del Norte

Antofagasta - Chile

\title{
M-FUZZIFYING BASES *
}

\author{
XIU XIN \\ and \\ $F U$-GUI SHI \\ BEIJING INSTITUTE OF TECHNOLOGY, CHINA \\ Received: October 2009. Accepted: October 2009
}

\begin{abstract}
In this paper, we continue the study of $M$-fuzzifying matroids. We define the notion of an $M$-fuzzifying base and discuss some properties of the dual matroids of basic $M$-fuzzifying matroids.
\end{abstract}

Keywords : $M$-fuzzifying bases; $M$-fuzzifying matroids; Dual matroids

Mathematics Subject Classification (2000) : 05B35, 52B40

\footnotetext{
${ }^{*}$ The project is supported by the National Natural Science Foundation of China (10971242)
} 


\section{Introduction}

In $[12,13]$, when $M$ is a complete lattice, Shi defined an $M$-fuzzifying matroid to be the pair $(E, \mathcal{I})$, where $\mathcal{I}$ is a map from $2^{E}$ to $M$ satisfying three axioms. Thus each subset of $E$ can be regarded as an independent set to some degree. Moreover, Shi defined the $M$-fuzzifying rank of a set $A$ as an $M$-fuzzy natural number $R(A): \mathbf{N} \rightarrow M$. $M$-fuzzifying matroids and $M$-fuzzifying rank functions are one-to-one corresponding. This paper treats the notion of $M$-fuzzifying bases. In subsequent papers we will deals with $M$-fuzzifying circuits and other fuzzy concepts related to $M$-fuzzifying matroids.

\section{Preliminaries}

We will use the following notation in establishing the results of this paper.

If $E$ is a finite set, $\mathcal{A} \subseteq 2^{E}$, define

$$
\begin{gathered}
\operatorname{Com}(\mathcal{A})=\{A \subseteq E: E-A \in \mathcal{A}\}, \\
\operatorname{Low}(\mathcal{A})=\{A \subseteq E: \exists B \in \mathcal{A}, A \subseteq B\}, \\
\operatorname{Max}(\mathcal{A})=\{A \in \mathcal{A}: \forall B \in \mathcal{A} \text {, if } A \subseteq B \text {, then } A=B\} .
\end{gathered}
$$

Throughout this paper, $M$ always denotes a completely distributive lattice and $M^{E}$ is the set of all $M$-fuzzy sets on $E$. The smallest element and the largest element in $M$ are denoted by $\perp$ and $T$, respectively. We often do not distinguish a crisp subset $A$ of $E$ and its characteristic function $\chi_{A}$.

An element $a$ in $M$ is called a prime element if $a \geq b \wedge c$ implies $a \geq b$ or $a \geq c$. $a$ in $M$ is called co-prime if $a \leq b \vee c$ implies $a \leq b$ or $a \leq c$ [2]. The set of non-unit prime elements in $M$ is denoted by $P(M)$. The set of non-zero co-prime elements in $M$ is denoted by $J(M)$.

The binary relation $\prec$ in $M$ is defined as follows: for $a, b \in M, a \prec b$ if and only if for every subset $D \subseteq M$, the relation $b \leq \sup D$ always implies the existence of $d \in D$ with $a \leq d$ [1]. $\{a \in M: a \prec b\}$ is called the greatest minimal family of $b$ in the sense of [14], denoted by $\beta(b)$, and $\beta^{*}(b)=\beta(b) \cap J(M)$. Moreover, for $b \in M$, we define $\alpha(b)=\{a \in M$ : $\left.a \prec^{o p} b\right\}$ and $\alpha^{*}(b)=\alpha(b) \cap P(M)$. In a completely distributive lattice $M$, $\alpha$ is an $\Lambda-\bigcup$ map, $\beta$ is a union-preserving map, and there exist $\alpha(b)$ and $\beta(b)$ for each $b \in M$ such that $b=\bigvee \beta(b)=\bigwedge \alpha(b)$ (see [14]). Note that $\beta(\perp)=\emptyset$ and $\alpha(\top)=\emptyset$. 
For any $A \in M^{E}$ and any $a \in M$, we define

$$
\begin{aligned}
& A_{[a]}=\{x \in E: A(x) \geq a\}, \quad A^{(a)}=\{x \in E: A(x) \not \leq a\}, \\
& A_{(a)}=\{x \in E: a \in \beta(A(x))\}, \quad A^{[a]}=\{x \in E: a \notin \alpha(A(x))\} .
\end{aligned}
$$

Some properties of these cut sets can be found in $[4,6,8,9,10,11]$.

In $[5,7,15]$, a crisp matroid and a crisp base for a crisp matroid are usually defined as follows:

Definition 2.1. Let $E$ be a finite set. $\mathcal{I} \subseteq 2^{E}$ is called a system of matroid independent sets on $E$ if it satisfies

(I1) $\emptyset \in \mathcal{I}$;

(I2) $A \in \mathcal{I}$ and $B \subseteq A$, then $B \in \mathcal{I}$;

(I3) For any $A, B \in \mathcal{I}$ which satisfy $|A|<|B|$, there exists $e \in B-A$ such that $A \cup\{e\} \in \mathcal{I}$, where $|A|,|B|$ denote the cardinality of $A, B$.

The set of all systems of matroid independent sets on $E$ is denoted by $\mathbf{I}(E)$ and $(E, \mathcal{I})$ is called a crisp matroid.

Definition 2.2. Let $E$ be a finite set. $A$ subset $\mathcal{B} \subseteq 2^{E}$ is called a crisp base on $E$ if it satisfies

(B1) $\mathcal{B} \neq \emptyset$;

(B2) If $B_{1}, B_{2} \in \mathcal{B}$ and $x \in B_{1}-B_{2}$, then $\left(B_{1}-\{x\}\right) \cup\{y\} \in \mathcal{B}$ for some $y \in B_{2}-B_{1}$.

The set of all crisp bases on $E$ is denoted by $\mathbf{B}(E)$.

Theorem 2.3. Let $E$ be a finite set. $A$ subset $\emptyset \neq \mathcal{B} \subseteq 2^{E}$ is a crisp base on $E$ if and only if it satisfies

$(\mathrm{B} 2)^{\prime}$ If $B_{1}, B_{2} \in \mathcal{B}$ and $x \in B_{1}-B_{2}$, then $\left(B_{2}-\{y\}\right) \cup\{x\} \in \mathcal{B}$ for some $y \in B_{2}-B_{1}$.

Theorem 2.4. (1) If $\mathcal{I} \in \mathbf{I}(E)$, then $\operatorname{Max}(\mathcal{I}) \in \mathbf{B}(E)$;

(2) If $\mathcal{B} \in \mathbf{B}(E)$, then $\operatorname{Low}(\mathcal{B}) \in \mathbf{I}(E)$;

(3) $\operatorname{Max} \circ \operatorname{Low}(\mathcal{B})=\mathcal{B}(\forall \mathcal{B} \in \mathbf{B}(E)) ; \operatorname{Low} \circ \operatorname{Max}(\mathcal{I})=\mathcal{I}(\forall \mathcal{I} \in \mathbf{I}(E))$.

Definition 2.5. Let $(E, \mathcal{I})$ be a crisp matroid. Define $\left(\mathcal{B}_{\mathcal{I}}\right)^{*}=\operatorname{Com}(\operatorname{Max}(\mathcal{I}))$ and $\mathcal{I}^{*}=\operatorname{Low}\left(\left(\mathcal{B}_{\mathcal{I}}\right)^{*}\right)$, then $\left(\mathcal{B}_{\mathcal{I}}\right)^{*}$ is a crisp base on $E$ and $\left(E, \mathcal{I}^{*}\right)$ is a crisp matroid. $\left(E, \mathcal{I}^{*}\right)$ is called the dual matroid of $(E, \mathcal{I})$.

Theorem 2.6. Let $(E, \mathcal{I})$ be a crisp matroid, for every $A \subseteq E$, then

(1) $R_{\mathcal{I}^{*}}(A)=|A|-R_{\mathcal{I}}(E)+R_{\mathcal{I}}(E-A)$;

(2) $R_{\mathcal{I}}(A)+R_{\mathcal{I}}(E-A)-R_{\mathcal{I}}(E)=R_{\mathcal{I}}(A)+R_{\mathcal{I}^{*}}(A)-|A|=R_{\mathcal{I}^{*}}(A)+$ $R_{\mathcal{I}^{*}}(E-A)-R_{\mathcal{I}^{*}}(E)$.

Where $R_{\mathcal{I}}$ and $R_{\mathcal{I}^{*}}$ denote the rank functions for $(E, \mathcal{I})$ and $\left(E, \mathcal{I}^{*}\right)$. 
In $[12,13]$, Shi defined an $M$-fuzzifying matroid as follows:

Definition 2.7. Let $E$ be a finite set. If a map $\mathcal{I}: 2^{E} \rightarrow M$ satisfies the following conditions:

(FI1) $\mathcal{I}(\emptyset)=\top$;

(FI2) For any $A, B \in 2^{E}, A \subseteq B \Rightarrow \mathcal{I}(A) \geq \mathcal{I}(B)$;

(FI3) If $A, B \in 2^{E}$ and $|A|<|B|$, then $\bigvee_{e \in B-A} \mathcal{I}(A \cup\{e\}) \geq \mathcal{I}(A) \wedge \mathcal{I}(B)$. Then the pair $(E, \mathcal{I})$ is called an $M$-fuzzifying matroid. $\mathcal{I}$ is called a fuzzy family of independent sets on $E$. For $A \in 2^{E}, \mathcal{I}(A)$ can be regarded as the degree of the set $A$ to be an independent set. $A[0,1]$-fuzzifying matroid is also called a fuzzifying matroid for short.

Theorem 2.8. Let $E$ be a finite set and $\mathcal{I}: 2^{E} \rightarrow M$ be a map. Then $(E, \mathcal{I})$ is an $M$-fuzzifying matroid if and only if for each $a \in J(M),\left(E, \mathcal{I}_{[a]}\right)$ is a crisp matroid.

Definition 2.9. Let $\mathbf{N}$ denote the set of all natural numbers. An $M$-fuzzy natural number is an antitone map $\lambda: \mathbf{N} \rightarrow M$ satisfying

$$
\lambda(0)=\top, \bigwedge_{n \in \mathbf{N}} \lambda(n)=\perp .
$$

The set of all $M$-fuzzy natural numbers is denoted by $\mathbf{N}(M)$.

Definition 2.10. For any $m \in \mathbf{N}$, define $\underline{m} \in \mathbf{N}(M)$ such that

$$
\underline{m}= \begin{cases}\top, & \text { if } \quad t \leq m, \\ \perp, & \text { if } \quad t \geq m+1 .\end{cases}
$$

Definition 2.11. For any $\lambda, \mu \in \mathbf{N}(M)$, define the addition $\lambda+\mu$ of $\lambda$ and $\mu$ as follows: for any $n \in \mathbf{N}$,

$$
(\lambda+\mu)(n)=\bigvee_{k+l=n}(\lambda(k) \wedge \mu(l)) .
$$

Theorem 2.12. For any $\lambda, \mu \in \mathbf{N}(M)$ and any $a \in J(M)$, it follows that

$$
(\lambda+\mu)_{[a]}=\lambda_{[a]}+\mu_{[a]} .
$$

Definition 2.13. Let $(E, \mathcal{I})$ be an $M$-fuzzifying matroid. The map $R_{\mathcal{I}}$ : $2^{E} \rightarrow \mathbf{N}(M)$ defined by

$$
R_{\mathcal{I}}(A)(n)=\bigvee\{\mathcal{I}(B): B \subseteq A,|B| \geq n\}
$$

is called the $M$-fuzzifying rank function for $(E, \mathcal{I})$. If $A \in 2^{E}$, then $R_{\mathcal{I}}(A)$ is called the $M$-fuzzifying rank of $A$. 
Theorem 2.14. Let $(E, \mathcal{I})$ be an $M$-fuzzifying matroid and $R_{\mathcal{I}}$ be the $M$ fuzzifying rank function for $(E, \mathcal{I})$. For each $a \in J(M)$, let $R_{\mathcal{I}_{[a]}}$ denote the rank function for $\left(E, \mathcal{I}_{[a]}\right)$. Then $R_{\mathcal{I}_{[a]}}(A)=R_{\mathcal{I}}(A)_{[a]}$ for each $A \in 2^{E}$.

\section{3. $M$-fuzzifying bases}

The obvious $M$-fuzzifying analog of a crisp base is the following:

Definition 3.1. Let $E$ be a finite set. $A$ map $\mathcal{B}: 2^{E} \rightarrow M$ is called an $M$-fuzzifying base on $E$ if it satisfies

(FB1) $\underset{B \in 2^{E}}{\bigvee} \mathcal{B}(B)=\top$;

(FB2) $\forall B_{1}, B_{2} \in 2^{E}, \bigwedge_{x \in B_{1}-B_{2}} \bigvee_{y \in B_{2}-B_{1}} \mathcal{B}\left(\left(B_{1}-\{x\}\right) \cup\{y\}\right) \geq \mathcal{B}\left(B_{1}\right) \wedge$ $\mathcal{B}\left(B_{2}\right)$.

$A[0,1]$-fuzzifying base is also called a fuzzifying base for short.

Example 3.2. Let $E=\{x, y\}$. Define $\mathcal{B}: 2^{E} \rightarrow[0,1]$ by

$$
\mathcal{B}(A)= \begin{cases}0, & A \in\{\emptyset,\{x, y\}\} ; \\ 1, & A=\{x\} ; \\ \frac{1}{2}, & A=\{y\} .\end{cases}
$$

Obviously, $\mathcal{B}$ is a fuzzifying base on $E$.

Theorem 3.3. Let $E$ be a finite set and $\mathcal{B}: 2^{E} \rightarrow M$ be a map. Then the following conditions are equivalent:

(1) $\mathcal{B}$ is an $M$-fuzzifying base on $E$;

(2) For each $a \in J(M), \mathcal{B}_{[a]}$ is a crisp base on $E$;

(3) For each $a \in P(M), \mathcal{B}^{(a)}$ is a crisp base on $E$.

Proof. $\quad(1) \Rightarrow(2)$. For each $a \in J(M)$. By (FB1), $\underset{B \in 2^{E}}{\bigvee} \mathcal{B}(B)=\top \geq a$, thus $\mathcal{B}(B) \geq a$ for some $B \in 2^{E}$, hence $\mathcal{B}_{[a]} \neq \emptyset$, i.e $\mathcal{B}_{[a]}$ satisfies (B1). Let $B_{1}, B_{2} \in \mathcal{B}_{[a]}$ and $x \in B_{1}-B_{2}$, then $\underset{y \in B_{2}-B_{1}}{\bigvee} \mathcal{B}\left(\left(B_{1}-\{x\}\right) \cup\{y\}\right) \geq$ $\mathcal{B}\left(B_{1}\right) \wedge \mathcal{B}\left(B_{2}\right) \geq a$ by (FB2). As $a \in J(M),\left(B_{1}-\{x\}\right) \cup\{y\} \in \mathcal{B}_{[a]}$ for some $y \in B_{2}-B_{1}$. This means that $\mathcal{B}_{[a]}$ satisfies (B2).

$(2) \Rightarrow(1)$. By $(2)$, for each $a \in J(M), \mathcal{B}(B) \geq a$ for some $B \in 2^{E}$, thus $\bigvee_{B \in 2^{E}} \mathcal{B}(B) \geq a$, hence $\bigvee_{B \in 2^{E}} \mathcal{B}(B)=\top$, i.e. (FB1) holds. Let $B_{1}, B_{2} \in 2^{E}$, $x \in B_{1}-B_{2}$, and $\mathcal{B}\left(B_{1}\right) \wedge \mathcal{B}\left(B_{2}\right) \neq \perp$. For every $\quad a \in J(M) \quad$ and 
$a \leq \mathcal{B}\left(B_{1}\right) \wedge \mathcal{B}\left(B_{2}\right)$, then $B_{1}, B_{2} \in \mathcal{B}_{[a]}$, thus there exists $y \in B_{2}-B_{1}$ such that $\left(B_{1}-\{x\}\right) \cup\{y\} \in \mathcal{B}_{[a]}$, hence $\underset{y \in B_{2}-B_{1}}{\bigvee} \mathcal{B}\left(\left(B_{1}-\{x\}\right) \cup\{y\}\right) \geq a$. Therefore, $\bigwedge_{x \in B_{1}-B_{2}} \underset{y \in B_{2}-B_{1}}{\bigvee} \mathcal{B}\left(\left(B_{1}-\{x\}\right) \cup\{y\}\right) \geq \mathcal{B}\left(B_{1}\right) \wedge \mathcal{B}\left(B_{2}\right)$.

$(1) \Rightarrow(3)$. For each $a \in P(M)$. By (FB1), $\underset{B \in 2^{E}}{\bigvee} \mathcal{B}(B)=\top \not \leq a$, thus $\mathcal{B}(B) \not \leq a$ for some $B \in 2^{E}$, hence $\mathcal{B}^{(a)} \neq \emptyset$, i.e $\mathcal{B}^{(a)}$ satisfies (B1). Let $B_{1}, B_{2} \in \mathcal{B}^{(a)}$ and $x \in B_{1}-B_{2}$, by (FB2) and $a \in P(M)$, we have $\bigvee_{y \in B_{2}-B_{1}} \mathcal{B}\left(\left(B_{1}-\{x\}\right) \cup\{y\}\right) \geq \mathcal{B}\left(B_{1}\right) \wedge \mathcal{B}\left(B_{2}\right) \not \leq a$, hence $\left(B_{1}-\{x\}\right) \cup\{y\} \in$ $\mathcal{B}^{(a)}$ for some $y \in B_{2}-B_{1}$. This means that $\mathcal{B}^{(a)}$ satisfies (B2).

$(3) \Rightarrow(1)$. By (3), for each $a \in P(M), B \in \mathcal{B}^{(a)}$ for some $B \in 2^{E}$, i.e. $\mathcal{B}(B) \not \leq a$ for some $B \in 2^{E}$, thus $\bigvee_{B \in 2^{E}} \mathcal{B}(B) \not \leq a$, hence $\underset{B \in 2^{E}}{\bigvee} \mathcal{B}(B)=\top$. Let $B_{1}, B_{2} \in 2^{E}, x \in B_{1}-B_{2}$, and $\mathcal{B}\left(B_{1}\right) \wedge \mathcal{B}\left(B_{2}\right) \neq \perp$. For every $a \in P(M)$ and $\mathcal{B}\left(B_{1}\right) \wedge \mathcal{B}\left(B_{2}\right) \not \leq a$, then $\mathcal{B}\left(B_{1}\right) \not \leq a$ and $\mathcal{B}\left(B_{2}\right) \not \leq a$, i.e. $B_{1}, B_{2} \in \mathcal{B}^{(a)}$, thus there exists $y \in B_{2}-B_{1}$ such that $\left(B_{1}-\{x\}\right) \cup\{y\} \in \mathcal{B}^{(a)}$, hence $\bigvee_{y \in B_{2}-B_{1}} \mathcal{B}\left(\left(B_{1}-\{x\}\right) \cup\{y\}\right) \not \leq a$. Therefore, $\bigwedge_{x \in B_{1}-B_{2}} \bigvee_{y \in B_{2}-B_{1}}^{\bigvee} \mathcal{B}\left(\left(B_{1}-\{x\}\right) \cup\right.$ $\{y\}) \geq \mathcal{B}\left(B_{1}\right) \wedge \mathcal{B}\left(B_{2}\right)$.

By Theorem 2.3 and Theorem 3.3, we can obtain the following theorem.

Theorem 3.4. Let $E$ be a finite set and $\mathcal{B}: 2^{E} \rightarrow M$ be a map. Then $\mathcal{B}$ is an $M$-fuzzifying base on $E$ if and only if it satisfies the following conditions:

(FB1) $\bigvee_{B \in 2^{E}} \mathcal{B}(B)=\top$; $\mathcal{B}\left(B_{2}\right)$.

$(\mathrm{FB} 2)^{\prime} \forall B_{1}, B_{2} \in 2^{E}, \underset{x \in B_{1}-B_{2}}{\wedge} \underset{y \in B_{2}-B_{1}}{\bigvee} \mathcal{B}\left(\left(B_{2}-\{y\}\right) \cup\{x\}\right) \geq \mathcal{B}\left(B_{1}\right) \wedge$

Theorem 3.5. Let $E$ be a finite set and $\mathcal{B}: 2^{E} \rightarrow M$ be a map. If $\alpha(a \vee b)=\alpha(a) \cap \alpha(b)$ for any $a, b \in M$, then the following conditions are equivalent:

(1) $\mathcal{B}$ is an $M$-fuzzifying base on $E$;

(2) For each $a \in M \backslash\{\top\}, \mathcal{B}^{[a]}$ is a crisp base on $E$;

(3) For each $a \in P(M), \mathcal{B}^{[a]}$ is a crisp base on $E$.

Proof. $\quad(1) \Rightarrow(2)$. For each $a \in M \backslash\{\top\}$. By (FB1), $\underset{B \in 2^{E}}{\bigvee} \mathcal{B}(B)=\top$, hence $a \notin \alpha(\top)=\alpha\left(\bigvee_{B \in 2^{E}} \mathcal{B}(B)\right)=\bigcap_{B \in 2^{E}} \alpha(\mathcal{B}(B))$, thus $a \notin \alpha(\mathcal{B}(B))$ for some $B \in 2^{E}$, i. e. $B \in \mathcal{B}^{[a]}$. This implies $\mathcal{B}^{[a]}$ satisfies (B1). Let 
$B_{1}, B_{2} \in \mathcal{B}^{[a]}$ and $x \in B_{1}-B_{2}$, then $a \notin \alpha\left(\mathcal{B}\left(B_{1}\right)\right) \cup \alpha\left(\mathcal{B}\left(B_{2}\right)\right)=\alpha\left(\mathcal{B}\left(B_{1}\right) \wedge\right.$ $\left.\mathcal{B}\left(B_{1}\right)\right)$ and $\underset{y \in B_{2}-B_{1}}{\bigvee} \mathcal{B}\left(\left(B_{1}-\{x\}\right) \cup\{y\}\right) \geq \mathcal{B}\left(B_{1}\right) \wedge \mathcal{B}\left(B_{2}\right)$ by (FB2), hence $a \notin \alpha\left(\bigvee_{y \in B_{2}-B_{1}} \mathcal{B}\left(\left(B_{1}-\{x\}\right) \cup\{y\}\right)\right)=\bigcap_{y \in B_{2}-B_{1}} \alpha\left(\mathcal{B}\left(\left(B_{1}-\{x\}\right) \cup\{y\}\right)\right)$, thus $a \notin \alpha\left(\left(\mathcal{B}\left(B_{1}-\{x\}\right) \cup\{y\}\right)\right)$ for some $y \in B_{2}-B_{1}$, i.e. $\left(B_{1}-\{x\}\right) \cup\{y\} \in$ $\mathcal{B}^{[a]}$ for some $y \in B_{2}-B_{1}$. This means that $\mathcal{B}^{[a]}$ satisfies (B2).

$(2) \Rightarrow(1)$. For each $a \in M \backslash\{\top\}$, by $\mathcal{B}^{[a]}$ satisfies (B1), then $a \notin$ $\alpha(\mathcal{B}(B))$ for some $B \in 2^{E}$, thus $a \notin \bigcap_{B \in 2^{E}} \alpha(\mathcal{B}(B))=\alpha\left(\bigvee_{B \in 2^{E}} \mathcal{B}(B)\right)$, hence $\bigvee_{B \in 2^{E}} \mathcal{B}(B)=\wedge \alpha\left(\bigvee_{B \in 2^{E}} \mathcal{B}(B)\right)=\top$, i.e. (FB1) holds. Let $B_{1}, B_{2} \in 2^{E}$, $x \in B_{1}-B_{2}$, and $\mathcal{B}\left(B_{1}\right) \wedge \mathcal{B}\left(B_{2}\right) \neq \perp$. For every $a \in M \backslash\{\top\}$ and $a \notin \alpha\left(\mathcal{B}\left(B_{1}\right) \wedge \mathcal{B}\left(B_{2}\right)\right)=\alpha\left(\mathcal{B}\left(B_{1}\right)\right) \cup \alpha\left(\mathcal{B}\left(B_{2}\right)\right)$, then $a \notin \alpha\left(\mathcal{B}\left(B_{1}\right)\right)$ and $a \notin \alpha\left(\mathcal{B}\left(B_{2}\right)\right)$, i.e. $B_{1}, B_{2} \in \mathcal{B}^{[a]}$, thus there exists $y \in B_{2}-B_{1}$ such that $\left(B_{1}-\{x\}\right) \cup\{y\} \in \mathcal{B}^{[a]}$, hence $a \notin \alpha\left(\mathcal{B}\left(\left(B_{1}-\{x\}\right) \cup\{y\}\right)\right), a \notin$ $\alpha\left(\bigvee_{y \in B_{2}-B_{1}} \mathcal{B}\left(\left(B_{1}-\{x\}\right) \cup\{y\}\right)\right)$. Therefore, $\bigwedge_{x \in B_{1}-B_{2}} \underset{y \in B_{2}-B_{1}}{\bigvee} \mathcal{B}\left(\left(B_{1}-\{x\}\right) \cup\right.$ $\{y\}) \geq \mathcal{B}\left(B_{1}\right) \wedge \mathcal{B}\left(B_{2}\right)$.

Analogously, we can obtain $(1) \Leftrightarrow(3)$.

Theorem 3.6. Let $E$ be a finite set and $\mathcal{B}: 2^{E} \rightarrow M$ be a map. If $\beta(a \wedge b)=\beta(a) \cap \beta(b)$ for any $a, b \in M$, then the following conditions are equivalent:

(1) $\mathcal{B}$ is an $M$-fuzzifying base on $E$;

(2) For each $a \in \beta(\top), \mathcal{B}_{(a)}$ is a crisp base on $E$.

Proof. $\quad(1) \Rightarrow(2)$. Suppose that $\mathcal{B}$ is an $M$-fuzzifying base on $E$. Then for any $a \in \beta(\top), a \in \beta\left(\bigvee_{B \in 2^{E}} \mathcal{B}(B)\right)=\bigcup_{B \in 2^{E}} \beta(\mathcal{B}(B))$ by (FB1), thus $a \in$ $\beta(\mathcal{B}(B))$ for some $B \in 2^{E}$, hence $B \in \mathcal{B}_{(a)}$, which means that $\mathcal{B}_{(a)}$ satisfies (B1). Let $B_{1}, B_{2} \in \mathcal{B}_{(a)}$ and $x \in B_{1}-B_{2}$, then $a \in \beta\left(\mathcal{B}\left(B_{1}\right)\right) \cap \beta\left(\mathcal{B}\left(B_{1}\right)\right)=$ $\beta\left(\mathcal{B}\left(B_{1}\right) \wedge \mathcal{B}\left(B_{2}\right)\right)$, and $\underset{y \in B_{2}-B_{1}}{\bigvee} \mathcal{B}\left(\left(B_{1}-\{x\}\right) \cup\{y\}\right) \geq \mathcal{B}\left(B_{1}\right) \wedge \mathcal{B}\left(B_{2}\right)$ by (FB2), hence $a \in \beta\left(\mathcal{B}\left(\left(B_{1}-\{x\}\right) \cup\{y\}\right)\right)$ for some $y \in B_{2}-B_{1}$, i.e. $\left(B_{1}-\{x\}\right) \cup\{y\} \in \mathcal{B}_{(a)}$ for some $y \in B_{2}-B_{1}$. This means that $\mathcal{B}_{(a)}$ satisfies (B2). 
$(2) \Rightarrow(1)$. By (B1), for each $a \in \beta(\top), B \in \mathcal{B}_{(a)}$ for some $B \in 2^{E}$, thus $a \in \beta(\mathcal{B}(B)) \subseteq \beta\left(\bigvee_{B \in 2^{E}} \mathcal{B}(B)\right)$, hence $\bigvee_{B \in 2^{E}} \mathcal{B}(B)=\bigvee \beta\left(\bigvee_{B \in 2^{E}} \mathcal{B}(B)\right) \geq$ $\bigvee_{a \in \beta(T)} a=\top$, i.e (FB1) holds. Let $B_{1}, B_{2} \in 2^{E}, \mathcal{B}\left(B_{1}\right) \wedge \mathcal{B}\left(B_{2}\right) \neq \perp$, $a \in \beta(T)$

$x \in B_{1}-B_{2}$, and $a \in \beta\left(\mathcal{B}\left(B_{1}\right) \wedge \mathcal{B}\left(B_{2}\right)\right)$. Then $a \in \beta\left(\mathcal{B}\left(B_{1}\right)\right)$ and $a \in$ $\beta\left(\mathcal{B}\left(B_{2}\right)\right)$, i.e. $B_{1}, B_{2} \in \mathcal{B}_{(a)}$. Hence there exists $y \in B_{2}-B_{1}$ such that $\left(B_{1}-\{x\}\right) \cup\{y\} \in \mathcal{B}_{(a)}$ by $\mathcal{B}_{(a)}$ is a crisp base on $E$. This shows that $a \in \beta\left(\mathcal{B}\left(\left(B_{1}-\{x\}\right) \cup\{y\}\right)\right) \subseteq \beta\left(\bigvee_{y \in B_{2}-B_{1}} \mathcal{B}\left(\left(B_{1}-\{x\}\right) \cup\{y\}\right)\right)$. Therefore, $\bigvee_{y \in B_{2}-B_{1}} \mathcal{B}\left(\left(B_{1}-\{x\}\right) \cup\{y\}\right) \geq \mathcal{B}\left(B_{1}\right) \wedge \mathcal{B}\left(B_{2}\right)$. This means that (FB2) holds.

Theorem 3.7. Let $E$ be a finite set, Then the following conditions are equivalent:

(1) $\mathcal{B}$ is a fuzzifying base on $E$;

(2) For each $a \in(0,1], \mathcal{B}_{[a]}$ is a crisp base on $E$;

(3) For each $a \in[0,1), \mathcal{B}_{(a)}$ is a crisp base on $E$.

\section{The relation between $M$-fuzzifying bases and $M$-fuzzifying matroids}

Definition 4.1. Let $(E, \mathcal{I})$ be an $M$-fuzzifying matroid on $E$, if it satisfies $\operatorname{Max}\left(\mathcal{I}_{[b]}\right) \subseteq \operatorname{Max}\left(\mathcal{I}_{[a]}\right)$ for every $a, b \in J(M)$ and $a \leq b$, then it is called a basic $M$-fuzzifying matroid.

Remark 4.2. Some $M$-fuzzifying matroids are not basic $M$-fuzzifying matroids. For example, let $E=\{x, y, z\}$ and $M=[0,1]$. Define $\mathcal{I}: 2^{E} \rightarrow M$ by

$$
\mathcal{I}(A)= \begin{cases}1, & A \in\{\emptyset,\{x\},\{y\}\} ; \\ \frac{1}{2}, & A \in\{\{x, y\}\} ; \\ \frac{1}{3}, & A \in\{\{z\},\{x, z\}\} ; \\ 0, & A \in\{\{y, z\}, E\}\end{cases}
$$

Then

$$
\mathcal{I}_{[r]}= \begin{cases}\{\emptyset,\{x\},\{y\},\{z\},\{x, y\},\{x, z\}\}, & r \in\left(0, \frac{1}{3}\right] ; \\ \{\emptyset,\{x\},\{y\},\{x, y\}\}, & r \in\left(\frac{1}{3}, \frac{1}{2}\right] ; \\ \{\emptyset,\{x\},\{y\}\}, & r \in\left(\frac{1}{2}, 1\right]\end{cases}
$$


Hence $(E, \mathcal{I})$ is an $M$-fuzzifying matroid, but it is not a basic $M$-fuzzifying matroid since $\operatorname{Max}\left(\mathcal{I}_{[1]}\right) \nsubseteq \operatorname{Max}\left(\mathcal{I}_{\left[\frac{1}{2}\right]}\right)$.

Theorem 4.3. Let $\mathcal{B}$ be an $M$-fuzzifying base on $E$. Define a map $\mathcal{I}_{\mathcal{B}}$ : $2^{E} \rightarrow M$ by

$$
\mathcal{I}_{\mathcal{B}}(A)=\bigvee_{A \subseteq B} \mathcal{B}(B)
$$

Then $\mathcal{I}_{\mathcal{B}}$ is a basic $M$-fuzzifying matroid on $E$.

Proof. For every $a \in J(M), A \in\left(\mathcal{I}_{\mathcal{B}}\right)_{[a]} \Leftrightarrow \underset{A \subseteq B}{\bigvee} \mathcal{B}(B) \geq a \Leftrightarrow A \subseteq B$ and $\mathcal{B}(B) \geq a$ for some $B \in 2^{E} \Leftrightarrow A \subseteq B$ and $B \in \mathcal{B}_{[a]}$ for some $B \in 2^{E} \Leftrightarrow A \in$ $\operatorname{Low}\left(\mathcal{B}_{[a]}\right)$. Therefore, $\left(\mathcal{I}_{\mathcal{B}}\right)_{[a]}=\operatorname{Low}\left(\mathcal{B}_{[a]}\right)$ and $\operatorname{Max}\left(\left(\mathcal{I}_{\mathcal{B}}\right)_{[a]}\right)=\mathcal{B}_{[a]}$. By Theorem 3.3, $\left(E,\left(\mathcal{I}_{\mathcal{B}}\right)_{[a]}\right)$ is a crisp matroid for every $a \in J(M)$. Therefore, $\mathcal{I}_{\mathcal{B}}$ is a basic $M$-fuzzifying matroid on $E$.

When $M$ is a boolean algebra, we have Theorems 4.4-4.6.

Theorem 4.4. Let $(E, \mathcal{I})$ be an $M$-fuzzifying matroid. Define a map $\mathcal{B}_{\mathcal{I}}$ : $2^{E} \rightarrow M$ by

$$
\mathcal{B}_{\mathcal{I}}(B)=\bigwedge_{B \subset A}(\mathcal{I}(A))^{\prime} \wedge \mathcal{I}(B)
$$

Then $\mathcal{B}_{\mathcal{I}}$ is an $M$-fuzzifying base.

Proof. $\quad \forall a \in J(M), B \in\left(\mathcal{B}_{\mathcal{I}}\right)_{[a]} \Leftrightarrow\left(\mathcal{B}_{\mathcal{I}}\right)(B) \geq a \Leftrightarrow(\mathcal{I}(A))^{\prime} \wedge \mathcal{I}(B) \geq a$ for every $A \supset B \Leftrightarrow \mathcal{I}(B) \geq a$ and $(\mathcal{I}(A))^{\prime} \geq a$ for every $A \supset B \Leftrightarrow$ $\mathcal{I}(B) \geq a$ and $\mathcal{I}(A) \geq a$ for every $A \supset B \Leftrightarrow B \in \mathcal{I}_{[a]}$ and $A \notin \mathcal{I}_{[a]}$ for every $A \supset B \Leftrightarrow B \in \operatorname{Max}\left(\mathcal{I}_{[a]}\right)$. Hence $\left(\mathcal{B}_{\mathcal{I}}\right)_{[a]}=\operatorname{Max}\left(\mathcal{I}_{[a]}\right)(\forall a \in J(M))$, and $\mathcal{B}_{\mathcal{I}}(B)=\bigvee\left\{a \in J(M): B \in \operatorname{Max}\left(\mathcal{I}_{[a]}\right)\right\}$. By Theorem 3.3, $\mathcal{B}_{\mathcal{I}}$ is an $M$-fuzzifying base.

Theorem 4.5. (1) For an $M$-fuzzifying matroid $(E, \mathcal{I})$, it follows that $\mathcal{I}_{\mathcal{B}_{\mathcal{I}}}=\mathcal{I}$

(2) For an $M$-fuzzifying base $\mathcal{B}$, it follows that $\mathcal{B}_{\mathcal{I}_{\mathcal{B}}}=\mathcal{B}$.

Proof. (1) For every $a \in J(M)$, by Theorem 4.3 and Theorem 4.4, $\left(\mathcal{I}_{\mathcal{B}_{\mathcal{I}}}\right)_{[a]}=\operatorname{Low}\left(\left(\mathcal{B}_{\mathcal{I}}\right)_{[a]}\right)=\operatorname{Low}\left(\operatorname{Max}\left(\mathcal{I}_{[a]}\right)\right)=\mathcal{I}_{[a]}$.

(2) For every $a \in J(M)$, by Theorem 4.3 and Theorem 4.4, $\left(\mathcal{B}_{\mathcal{I}_{\mathcal{B}}}\right)_{[a]}=$ $\operatorname{Max}\left(\left(\mathcal{I}_{\mathcal{B}}\right)_{[a]}\right)=\operatorname{Max}\left(\operatorname{Low}\left(\mathcal{B}_{[a]}\right)\right)=\mathcal{B}_{[a]}$.

By Theorems 4.3-4.5, we can obtain the following two results: 
Theorem 4.6. There is a one-to-one correspondence between $M$-fuzzifying matroids and $M$-fuzzifying bases. That is, an $M$-fuzzifying matroid can be completely characterized by an $M$-fuzzifying base.

Remark 4.7. When $M$ is a boolean algebra, an $M$-fuzzifying matroid is equivalent to a basic $M$-fuzzifying matroid.

When $M=[0,1]$, we have Theorems 4.8-4.10.

Theorem 4.8. Let $(E, \mathcal{I})$ be a basic fuzzifying matroid. Define a map $\mathcal{B}_{\mathcal{I}}: 2^{E} \rightarrow[0,1]$ by

$$
\mathcal{B}_{\mathcal{I}}(B)=\bigvee\left\{a \in(0,1]: B \in \operatorname{Max}\left(\mathcal{I}_{[a]}\right)\right\}
$$

Then $\mathcal{B}_{\mathcal{I}}$ is a fuzzifying base on $E$.

Proof. For each $a \in(0,1]$, obviously, $\operatorname{Max}\left(\mathcal{I}_{[a]}\right) \subseteq\left(\mathcal{B}_{\mathcal{I}}\right)_{[a]} . \quad B \in$ $\left(\mathcal{B}_{\mathcal{I}}\right)_{[a]} \Leftrightarrow \bigvee\left\{a \in(0,1]: B \in \operatorname{Max}\left(\mathcal{I}_{[a]}\right)\right\} \geq a \Leftrightarrow a \leq b$ for some $b \in$ $(0,1]$ and $B \in \operatorname{Max}\left(\mathcal{I}_{[b]}\right)$. As $(E, \mathcal{I})$ is a basic fuzzifying matroid, $B \in$ $\operatorname{Max}\left(\mathcal{I}_{[b]}\right) \subseteq \operatorname{Max}\left(\mathcal{I}_{[a]}\right)$. This means that $\left(\mathcal{B}_{\mathcal{I}}\right)_{[a]} \subseteq \operatorname{Max}\left(\mathcal{I}_{[a]}\right)$. Therefore, $\left(\mathcal{B}_{\mathcal{I}}\right)_{[a]}=\operatorname{Max}\left(\mathcal{I}_{[a]}\right)$. By Theorem 3.3, $\mathcal{B}_{\mathcal{I}}$ is a fuzzifying base on $E$.

Theorem 4.9. (1) For a basic fuzzifying matroid $(E, \mathcal{I})$, it follows that $\mathcal{I}_{\mathcal{B}_{\mathcal{I}}}=\mathcal{I}$

(2) For a fuzzifying base $\mathcal{B}$, it follows that $\mathcal{B}_{\mathcal{I}_{\mathcal{B}}}=\mathcal{B}$.

Proof. (1) For every $a \in(0,1]$, by Theorem 4.3 and Theorem 4.8, $\left(\mathcal{I}_{\mathcal{B}_{\mathcal{I}}}\right)_{[a]}=\operatorname{Low}\left(\left(\mathcal{B}_{\mathcal{I}}\right)_{[a]}\right)=\operatorname{Low}\left(\operatorname{Max}\left(\mathcal{I}_{[a]}\right)\right)=\mathcal{I}_{[a]}$.

(2) For every $a \in(0,1]$, by Theorem 4.3 and Theorem 4.8, $\left(\mathcal{B}_{\mathcal{I}_{\mathcal{B}}}\right)_{[a]}=$ $\operatorname{Max}\left(\left(\mathcal{I}_{\mathcal{B}}\right)_{[a]}\right)=\operatorname{Max}\left(\operatorname{Low}\left(\mathcal{B}_{[a]}\right)\right)=\mathcal{I}_{[a]}$.

By Theorem 4.3, Theorem 4.8 and Theorem 4.9, we can obtain the following result:

Theorem 4.10. There is a one-to-one correspondence between basic fuzzifying matroids and fuzzifying bases. That is, a basic fuzzifying matroid can be completely characterized by a fuzzifying base. 


\section{The dual matroids of basic $M$-fuzzifying matroids}

In this section, $M$ is a boolean algebra or $M=[0,1]$.

Definition 5.1. Let $(E, \mathcal{I})$ be a basic $M$-fuzzifying matroid. Define a map $\left(\mathcal{B}_{\mathcal{I}}\right)^{*}: 2^{E} \rightarrow M$ by $\left(\mathcal{B}_{\mathcal{I}}\right)^{*}(B)=\mathcal{B}_{\mathcal{I}}(E-B)$ and let $\mathcal{I}^{*}=\mathcal{I}_{\left(\mathcal{B}_{\mathcal{I}}\right)^{*}}$, then $\left(\mathcal{B}_{\mathcal{I}}\right)^{*}$ is an $M$-fuzzifying base on $E$ and $\left(E, \mathcal{I}^{*}\right)$ is a basic $M$-fuzzifying matroid. $\left(E, \mathcal{I}^{*}\right)$ is called the dual matroid of $(E, \mathcal{I})$.

Proof. By the definition of $\left(\mathcal{B}_{\mathcal{I}}\right)^{*},\left(\mathcal{B}_{\mathcal{I}}\right)_{[a]}^{*}=\operatorname{Com}\left(\left(\mathcal{B}_{\mathcal{I}}\right)_{[a]}\right)$ for every $a \in J(M)$. Therefore, for every $a \in J(M),\left(\mathcal{B}_{\mathcal{I}}\right)_{[a]}^{*}$ is a crisp base on $E$. By Theorem $3.3,\left(\mathcal{B}_{\mathcal{I}}\right)^{*}$ is an $M$-fuzzifying base on $E$. By Theorem $4.3,\left(E, \mathcal{I}^{*}\right)$ is a basic $M$-fuzzifying matroid.

Theorem 5.2. Let $(E, \mathcal{I})$ be a basic $M$-fuzzifying matroid, then

(1) $\left(\mathcal{I}^{*}\right)^{*}=\mathcal{I}$;

(2) $\operatorname{Com}\left(\left(\mathcal{B}_{\mathcal{I}}\right)_{[a]}\right)=\left(\mathcal{B}_{\mathcal{I}^{*}}\right)_{[a]}$ for every $a \in J(M)$;

(3) $\mathcal{I}_{[a]}^{*}=\left(\mathcal{I}_{[a]}\right)^{*}$ for every $a \in J(M)$.

Proof. (1) By the definition of $\mathcal{I}^{*}$, Theorem 4.5 and Theorem 4.9, $\left(\mathcal{I}^{*}\right)^{*}=\left(\mathcal{I}_{\left(\mathcal{B}_{\mathcal{I}}\right)^{*}}\right)^{*}=\mathcal{I}_{\left(\mathcal{B}_{\mathcal{I}_{\left(\mathcal{B}_{\mathcal{I}}\right)^{*}}}\right)^{*}}=\mathcal{I}_{\left(\left(\mathcal{B}_{\left.\mathcal{I})^{*}\right)^{*}}\right.\right.}=\mathcal{I}_{\mathcal{B}_{\mathcal{I}}}=\mathcal{I}$.

(2) By Theorem 4.5, Theorem 4.9 and the definition of $\left(\mathcal{B}_{\mathcal{I}}\right)^{*}$, for every $a \in J(M),\left(\mathcal{B}_{\mathcal{I}^{*}}\right)_{[a]}=\left(\mathcal{B}_{\mathcal{I}}\right)_{[a]}^{*}=\operatorname{Com}\left(\left(\mathcal{B}_{\mathcal{I}}\right)_{[a]}\right)$.

(3) For every $a \in J(M), A \in \mathcal{I}_{[a]}^{*} \Leftrightarrow A \in \operatorname{Low}\left(\left(\mathcal{B}_{\mathcal{I}}\right)_{[a]}^{*}\right) \Leftrightarrow A \in$ $\operatorname{Low}\left(\operatorname{Com}\left(\left(\mathcal{B}_{\mathcal{I}}\right)_{[a]}\right)\right) \Leftrightarrow A \in \operatorname{Low}\left(\operatorname{Com}\left(\operatorname{Max}\left(\mathcal{I}_{[a]}\right)\right) \Leftrightarrow A \in\left(\mathcal{I}_{[a]}\right)^{*}\right.$.

Theorem 5.3. Let $(E, \mathcal{I})$ be a basic $M$-fuzzifying matroid, for every $A \subseteq$ $E$, then

(1) $R_{\mathcal{I}^{*}}(A)+R_{\mathcal{I}}(E)=|A|+R_{\mathcal{I}}(E-A)$;

(2) $R_{\mathcal{I}}(A)+R_{\mathcal{I}^{*}}(E)=\underline{\overline{|A|}}+R_{\mathcal{I}^{*}}(E-A)$.

Proof. $\quad(1) \forall a \in J(M),\left(R_{\mathcal{I}^{*}}(A)+R_{\mathcal{I}}(E)\right)_{[a]}=\left(R_{\mathcal{I}^{*}}(A)\right)_{[a]}+\left(R_{\mathcal{I}}(E)\right)_{[a]}=$ $R_{\mathcal{I}_{[a]}^{*}}(A)+R_{\mathcal{I}_{[a]}}(E)=R_{\left(\mathcal{I}_{[a]}\right)}(A)+R_{\mathcal{I}_{[a]}}(E)=|A|+R_{\mathcal{I}_{[a]}}(E-A)=$ $\left.\underline{(|A|)_{[a]}}+R_{\mathcal{I}}(E-A)\right)_{[a]}=\left(\underline{|A|}+R_{\mathcal{I}}(E-A)\right)_{[a]}$.

(2) By $(1)$ and Theorem $5.2(1), R_{\mathcal{I}}(A)+R_{\mathcal{I}^{*}}(E)=R_{\left(\mathcal{I}^{*}\right)^{*}}(A)+R_{\mathcal{I}^{*}}(E)=$ $\underline{|A|}+R_{\mathcal{I}^{*}}(E-A)$. 


\section{References}

[1] P. Dwinger, Characterizations of the complete homomorphic images of a completely distributive complete lattice I, Indagationes Mathematicae (Proceedings) 85, pp. 403-414, (1982).

[2] G. Gierz, et al., Continuous Lattices and Domains, Cambridge University Press, Cambridge, (2003).

[3] R. Goetschel, W. Voxman, Bases of fuzzy matroids, Fuzzy Sets and Systems 31, pp. 253-261, (1989).

[4] H.-L. Huang, F.-G. Shi, M-fuzzy numbers and their properties, Information Sciences 178, pp. 1141-1151, (2008).

[5] H.-J. Lai, Matroid Theory, Higher Education Press, Beijing (in Chinese), (2002).

[6] C. V. Negoita, D. A. Ralescu, Applications of Fuzzy Sets to Systems Analysis, Interdisciplinary Systems Research Series, vol. 11, Birkhaeuser, Basel, Stuttgart and Halsted Press, New York, (1975).

[7] J. G. Oxley, Matroid Theory, Oxford University Press, New York, (1992).

[8] F.-G. Shi, Theory of $L_{\beta}$-nested sets and $L_{\alpha}$-nested sets and its applications, Fuzzy Systems and Mathematics 4, pp. 65-72 (in Chinese), (1995).

[9] F.-G. Shi, M-fuzzy sets and prime element nested sets, J. Mathematical Research and Exposition 16, pp. 398-402 (in Chinese), (1996).

[10] F.-G. Shi, Theory of molecular nested sets and its applications, J. Yantai Teachers University (Natural Science) 1, pp. 33-36 (in Chinese), (1996).

[11] F.-G. Shi, $M$-fuzzy relation and $M$-fuzzy subgroup, J. Fuzzy Mathematics 8, pp. 491-499, (2000).

[12] F.-G. Shi, A new approach to the fuzzification of matroids, Fuzzy Sets and Systems 160, pp. 696-705, (2009). 
[13] F.-G. Shi, (L,M)-fuzzy matroids, Fuzzy Sets and Systems 160, pp. 2387-2400, (2009).

[14] G.-J. Wang, Theory of topological molecular lattices, Fuzzy Sets and Systems 47, pp. 351-376, (1992).

[15] D. J. A. Welsh, Matroid Theory, Oxford University Press, New York, (1976).

\section{Xiu Xin}

Department of Mathematics

Beijing Institute of Technology

Beijing, 100081,

P. R. China

e-mail : xinxiu518@163.com;

and

\section{Fu-Gui Shi}

Department of Mathematics

Beijing Institute of Technology

Beijing, 100081,

P. R. China

e-mail : fuguishi@bit.edu.cn 